\title{
CONVERSION BETWEEN DCT COEFFICIENTS AND IT COEFFICIENTS IN THE COMPRESSED DOMAIN FOR H.263 TO H.264 VIDEO TRANSCODING
}

\author{
Kai-Tat Fung and Wan-Chi Siu \\ Centre for Multimedia Signal Processing \\ Department of Electronic and Information Engineering \\ The Hong Kong Polytechnic University, Hung Hom, Kowloon, Hong Kong
}

\begin{abstract}
For transcoding a video sequence from the H.263 format to the H.264 format, it is beneficial to reuse as much information as possible in the original sequence. However, given the significant differences between the H.263 and the H.264 algorithms, transcoding is much more complex. Motivated by this, a set of operators is derived for converting the DCT coefficients to Integer Transform(IT) coefficients in the compressed domain. Using these operators, the proposed architecture is able to transcode the DCT coefficients to the Integer Transform coefficients directly. In other words, no complete decoding and reencoding processes are required. To further speed up the transcoding process, an approximation form of the DCT coefficients is proposed. Experimental results show that the proposed video transcoder can reduce substantially the amount of computation and provide transcoded videos with better quality as compared with those obtained from the conventional cascaded video transcoder. The proposed video transcoder can combine any existing fast searching algorithm for multiple reference frames and variable block sizes estimation to speed up the transcoding process.
\end{abstract}

\section{INTRODUCTION}

The H.264 video coding standard has been filed recently[1]. This new generation video standard, with its significant bandwidth savings, is expected to replace the H.263 for video compression in digital video systems[2]. Compared with the H.263 video, the H.264 video format can provide equivalent video at $1 / 3$ to $1 / 2$ of the $H .263$ bitrates. In spite of its complexity, the actual bandwidth savings compared to the H.263 provides a big motivation to migrate to H.264 video coding. The standard is intended for use in low bitrate applications including mobile phones, moderate bitrate applications such as video conferencing, and high bitrate applications such as digital TV. The complete migration to the new video coding algorithm will take several years since H.263 and MPEG are widely used in many multimedia applications nowadays. This creates an important need for transcoding technologies[3-8] that convert the widely available H.263 compressed videos to H.264 compressed format and vice versa. However, given the significant differences between the H.263 and the H.264 algorithms, transcoding is much more complex. One important issue in the transcoding of a video from the MPEG or H.263 format to the H.264/AVC format is to convert the DCT coefficients to the Integer Transform(IT) coefficients. Figure 1 shows the diagram of DCT coefficients to integer transform coefficients conversion for intra frame video transcoding. Figure 2 shows the conventional approach for transcoding the transformed coefficients in the pixel domain. The input is an $8 \times 8$ block of 2D-DCT coefficients, A. An inverse DCT is applied to A to recover an $8 \times 8$ pixel block. The $8 \times 8$ pixel block is divided into four $4 \times 4$ blocks (pixel domain data $i=0,1,2,3$ ). Each of the four blocks is passed to a corresponding integer transform to generate four $4 \times 4$ blocks of transform coefficients. These four blocks of transform coefficients are combined to form a single $8 \times 8$ block. This process introduces high computational complexity as well as reencoding errors. It is desired to perform the transcoding in the compressed domain to avoid the complete decoding and re-encoding process. In the next section, a set of operators are derived to transcode the DCT coefficients to Integer transform coefficients in the transform domain. Also, a fast approximation of the DCT coefficients is proposed.

The organization of this paper is as follows. Section II presents the formulation of the proposed operators and its fast approximation for H.263 to H.264 video transcoding. Experimental results are then given in Section III. Finally, some concluding remarks are provided in Section IV.

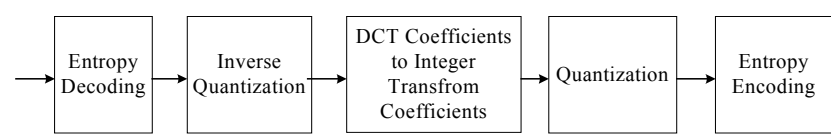

Figure 1. Intra block transcoding from H.263 to H.264/AVC 


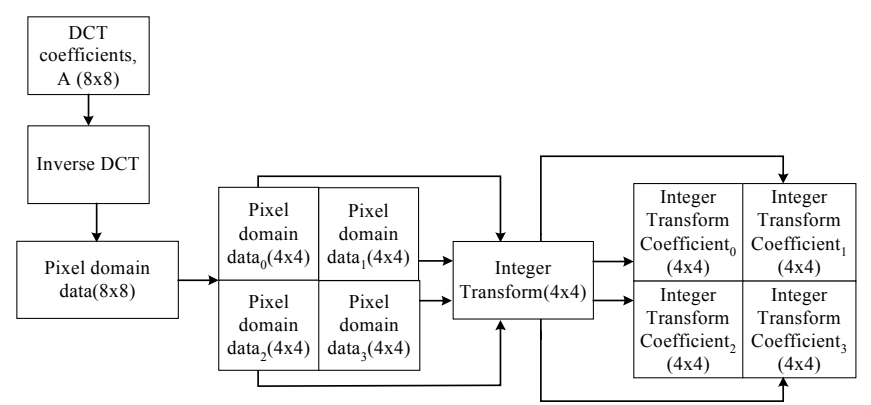

Figure 2. Conventional approach for transcoding the transformed coefficients in the pixel domain.

\section{DISCRETE COSINE TRANSFORM COEFFICIENTS TO INTEGER TRANSFORM COEFFICIENTS}

Let us recall the definition of the 2D-DCT of an $8 \times 8$ block, $\mathrm{A}$, as

$$
\operatorname{DCT}(A)=\hat{A}=T A T^{t}
$$

where $\mathrm{T}$ is an $8 \mathrm{x} 8 \mathrm{DCT}$ matrix with entries $\mathrm{t}(\mathrm{i}, \mathrm{j})$ given by

$$
t(i, j)=\frac{1}{2} k(i) \cos \frac{(2 j+1) i \pi}{16}
$$

$\mathrm{i}$ represents the row index, $\mathrm{j}$ represents the column index and

$$
k(i)=\left\{\begin{array}{cc}
\frac{1}{\sqrt{2}}, & i=0 \\
1, & \text { otherwise. }
\end{array}\right.
$$

The Integer Transform(IT) of a $4 \times 4$ block, $B$, can be defined as

$$
\mathrm{I}(\mathrm{B})=\mathrm{IBI}^{\mathrm{t}} \otimes(\text { scaling factor } 1)
$$

where I is a $4 \times 4$ IT matrix given by

$$
I=\left[\begin{array}{cccc}
1 & 1 & 1 & 1 \\
2 & 1 & -1 & -2 \\
1 & -1 & -1 & 1 \\
1 & -2 & 2 & -1
\end{array}\right]
$$

and scaling factor $1=\left[\begin{array}{cccc}a^{2} & a b / 2 & a^{2} & a b / 2 \\ a b / 2 & b^{2} / 4 & a b / 2 & b^{2} / 4 \\ a^{2} & a b / 2 & a^{2} & a b / 2 \\ a b / 2 & b^{2} / 4 & a b / 2 & b^{2} / 4\end{array}\right]$

$$
=\left[\begin{array}{cccc}
0.25 & 0.158 & 0.25 & 0.158 \\
0.158 & 0.1 & 0.158 & 0.1 \\
0.25 & 0.158 & 0.25 & 0.158 \\
0.158 & 0.1 & 0.158 & 0.1
\end{array}\right]
$$

$\mathrm{a}=0.5, b=\sqrt{\frac{2}{5}}$

where $\otimes$ represents the term by term multiplication.

Similarly, the inverse transform is given by:

$\mathrm{B}^{\prime}=\mathrm{J}[\mathrm{I}(\mathrm{B}) \otimes$ scaling factor 2$] \mathrm{J}^{\mathrm{t}}$

where $\mathrm{J}$ is a $4 \mathrm{x} 4$ inverse IT matrix given by

$$
J=\left[\begin{array}{cccc}
1 & 1 & 1 & 0.5 \\
1 & 0.5 & -1 & -1 \\
1 & -0.5 & -1 & 1 \\
1 & -1 & 1 & -0.5
\end{array}\right]
$$

and scaling factor $2=\left[\begin{array}{cccc}a^{2} & a b & a^{2} & a b \\ a b & b^{2} & a b & b^{2} \\ a^{2} & a b & a^{2} & a b \\ a b & b^{2} & a b & b^{2}\end{array}\right]$

$$
=\left[\begin{array}{cccc}
0.25 & 0.316 & 0.25 & 0.316 \\
0.316 & 0.4 & 0.316 & 0.4 \\
0.25 & 0.316 & 0.25 & 0.316 \\
0.316 & 0.4 & 0.316 & 0.4
\end{array}\right]
$$

In the following, we will derive a set of equations to transform the DCT coefficients, DCT(A), into the integer transform coefficients directly in the compressed domain. Similar formulation can be obtained for transcoding the integer transform coefficients to DCT coefficients.

Applying the inverse DCT to the DCT coefficients, DCT(A), we have

$$
\text { pixel domain data }=\mathrm{T}^{\mathrm{t}}[\mathrm{DCT}(\mathrm{A})] \mathrm{T}
$$

In order to satisfy the H.264 format, let us divide this reconstructed result into four groups. These four groups of data in the pixel domain can be extracted by using the proposed operators $\mathrm{S}_{\mathrm{i} 1}$ and $\mathrm{S}_{\mathrm{i} 2}$, i.e., we have

Pixel domain data $\mathrm{i}_{\mathrm{i}}=\mathrm{S}_{\mathrm{i} 1} \mathrm{~T}^{\mathrm{t}}\left[\mathrm{DCT}(\mathrm{A}) \mathrm{TS}_{\mathrm{i} 2}\right.$ for $\mathrm{i}=0,1,2,3$

$$
\begin{aligned}
& \text { where } s_{01}=s_{11}=s_{02}{ }^{T}=s_{22}{ }^{T}=\left[\begin{array}{llllllll}
1 & 0 & 0 & 0 & 0 & 0 & 0 & 0 \\
0 & 1 & 0 & 0 & 0 & 0 & 0 & 0 \\
0 & 0 & 1 & 0 & 0 & 0 & 0 & 0 \\
0 & 0 & 0 & 1 & 0 & 0 & 0 & 0
\end{array}\right] \text {, } \\
& s_{21}=s_{31}=s_{12}{ }^{T}=s_{32}{ }^{T}=\left[\begin{array}{llllllll}
0 & 0 & 0 & 0 & 1 & 0 & 0 & 0 \\
0 & 0 & 0 & 0 & 0 & 1 & 0 & 0 \\
0 & 0 & 0 & 0 & 0 & 0 & 1 & 0 \\
0 & 0 & 0 & 0 & 0 & 0 & 0 & 1
\end{array}\right]
\end{aligned}
$$


and $\mathrm{i}$ represents the index of the 4 groups of the integer transform coefficients.

Note that $\mathrm{A}$ is with a size of $8 \times 8$ and the integer transform coefficients is $4 \times 4$. Therefore, one set of DCT coefficients will give four sets of the integer transform coefficients.

Then we can apply the integer transform to the pixel domain data $_{\mathrm{i}}$. We have

Integer transform coefficients $_{\mathrm{i}}=\mathrm{I}$ (pixel domain data $\mathrm{d}_{\mathrm{i}} \mathrm{I}^{\mathrm{t}} \otimes$

(scaling factor 1)

Combining equations (4-6), we have

Integer transform coefficients $\mathrm{s}_{\mathrm{i}}=\mathrm{I}\left(\mathrm{S}_{\mathrm{i} 1} \mathrm{~T}^{\mathrm{t}} \mathrm{DCT}(\mathrm{A}) \mathrm{TS}_{\mathrm{i} 2}\right) \mathrm{I}^{\mathrm{t}} \otimes$

(scaling factor 1 )

Let us define the pre-computed operators, $\mathrm{P}_{1 \mathrm{i}}=\mathrm{I}^{*} \mathrm{~S}_{\mathrm{i} 1} * \mathrm{~T}^{\mathrm{t}}$ and $\mathrm{P}_{2 \mathrm{i}}=\mathrm{T} * \mathrm{~S}_{\mathrm{i} 2} * \mathrm{I}^{\mathrm{t}}$,

Therefore, we have

Integer transform coefficients ${ }_{\mathrm{i}}=\mathrm{P}_{1 \mathrm{i}} *[\mathrm{DCT}(\mathrm{A})]^{*} \mathrm{P}_{2 \mathrm{i}} \otimes$

where

$\mathrm{P}_{10}=\mathrm{P}_{11}=\left[\begin{array}{cccccccc}1.4142 & 1.2815 & 0 & -0.4500 & 0 & 0.3007 & 0 & -0.2549 \\ 0 & 0.9236 & 2.2304 & 1.7799 & 0 & -0.8638 & -0.1585 & 0.4824 \\ 0 & -0.1056 & 0 & 0.7259 & 1.4142 & 1.0864 & 0 & -0.5308 \\ 0 & 0.1169 & 0.1585 & -0.0922 & 0 & 1.0379 & 2.2304 & 1.9750\end{array}\right]$,

$\mathrm{P}_{12}=\mathrm{P}_{13}=\left[\begin{array}{llllllll}1.4142 & -1.2815 & 0 & 0.4500 & 0 & -0.3007 & 0 & 0.2549\end{array}\right]$, $\left.\begin{array}{llllllll}0 & 0.9236 & -2.2304 & 1.7799 & 0 & -0.8638 & 0.1585 & 0.4824\end{array}\right]$

$\begin{array}{llllllll}0 & 0.1056 & 0 & -0.7259 & 1.4142 & -1.0864 & 0 & 0.5308\end{array}$

$\begin{array}{llllllll}0 & 0.1169 & -0.1585 & -0.0922 & 0 & 1.0379 & -2.2304 & 1.9750\end{array}$

$\mathrm{P}_{20}=\mathrm{P}_{22}=\left[\begin{array}{cccc}1.4142 & 0 & 0 & 0 \\ 1.2815 & 0.9236 & -0.1056 & 0.1169 \\ 0 & 2.2304 & 0 & 0.1585 \\ -0.4500 & 1.7799 & 0.7259 & -0.0922 \\ 0 & 0 & 1.4142 & 0 \\ 0.3007 & -0.8638 & 1.0864 & 1.0379 \\ 0 & -0.1585 & 0 & 2.2304 \\ -0.2549 & 0.4824 & -0.5308 & 1.9750\end{array}\right]$

and

$$
\mathrm{P}_{21}=\mathrm{P}_{23}=\left[\begin{array}{cccc}
1.4142 & 0 & 0 & 0 \\
-1.2815 & 0.9236 & 0.1056 & 0.1169 \\
0 & -2.2304 & 0 & -0.1585 \\
0.4500 & 1.7799 & -0.7259 & -0.0922 \\
0 & 0 & 1.4142 & 0 \\
-0.3007 & -0.8638 & -1.0864 & 1.0379 \\
0 & 0.1585 & 0 & -2.2304 \\
0.2549 & 0.4824 & 0.5308 & 1.9750
\end{array}\right]
$$

Note that $\mathrm{P}_{1 \mathrm{i}}$ and $\mathrm{P}_{2 \mathrm{i}}$ can be pre-computed. Therefore, low computational complexity can be achieved since the transformation is done in the compressed domain. In other words, no complete decoding and re-encoding process are required to obtain the integer transform coefficients from DCT coefficients. To further speed up the transcoding process, an approximation form of the DCT coefficients is introduced. Since the energy distribution of the DCT blocks obtained from an incoming bitstream mainly concentrate on the low frequency region, it is beneficial to approximate the DCT coefficients using only a few significant DCT coefficients. The pattern of significant DCT coefficients for transcoding can be obtained by using the following equation which defines the energy of the DCT coefficients of a block with size $\mathrm{NxN}$,

$$
\begin{aligned}
& \text { energy }=\sum_{l=0}^{N-1} \sum_{m=0}^{N-1} B^{2}(l, m) \\
& \text { Approx. energy }=\sum_{l=0}^{j} \sum_{m=0}^{k} B^{2}(l, m)
\end{aligned}
$$

where $B(l, m)$ represents the $l^{\text {th }}$ row and $m^{\text {th }}$ column of the DCT coefficients. $\mathrm{j}$ and $\mathrm{k}$ represent the numbers of rows and columns to approximate the original DCT coefficients. Initially, $\mathrm{j}$ and $\mathrm{k}$ are set to zero. If the approximated energy is less than 0.95 time of the original energy, $\mathrm{j}$ or $\mathrm{k}$ will be increased by 1 until the desired approximation of the DCT coefficients is obtained. Note that the threshold 0.95 can be changed depending upon the application and the processing power of the transcoder. Using the above formulation, the architecture of the proposed transcoder is as shown in Figure 3. In Figure 3, the video transcoder performs an entropy decoding to extract the header information, coding mode, motion vectors and quantized DCT coefficients from the incoming bitstream. The motion vectors information will be used to speed up the motion vector re-estimation process. The DCT coefficients will be transcoded into the integer transform coefficients directly in the compressed domain using the proposed operators. Performance of the proposed approximation of DCT coefficients is given in the next section.

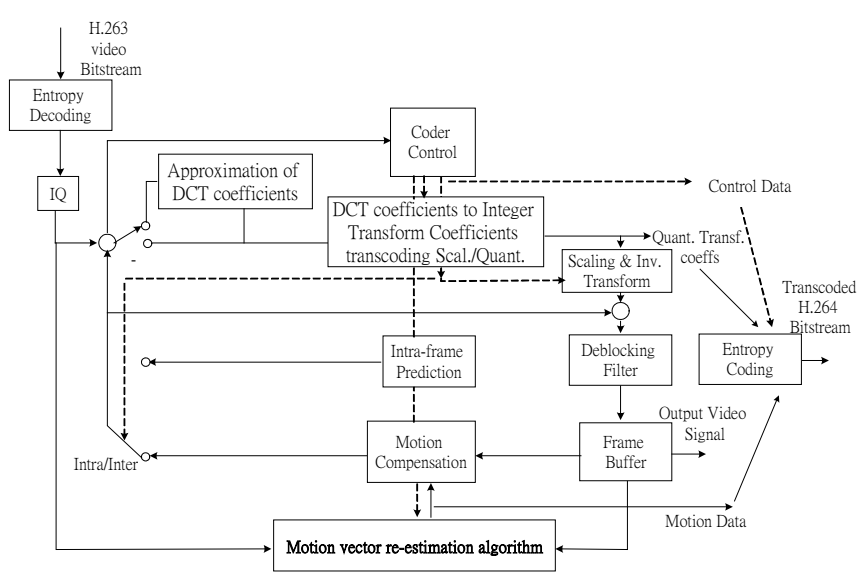

Figure 3. The proposed compressed domain video transcoder for H.263 to H.264

\section{EXPERIMENTAL RESULTS}

Extensive simulations and performance comparison have been done with reference to a cascaded pixel-domain transcoder. The cascaded transcoder was implemented 
using an H.263 video codec and an H.264 video codec. In the front encoder, the first frame was encoded as an intraframe (I-frame), and the remaining frames were encoded as interframes (P-frames). Results of our experimental work show that the proposed video transcoders outperform the conventional cascaded video transcoder in terms of computational complexity as shown in Table 1. The quality degradation was measured by using the approximation of DCT coefficients in the proposed video transcoder as compared with the conventional cascaded video transcoder. The speed up ratio is defined as the time for transcoding integer transform coefficients using the proposed transcoder with the approximation of DCT coefficients as compared with the time for conventional cascaded video transcoder. Theoretically, the speed up ratio for transcoding the DCT coefficients to Integer transform coefficients is about 2.8 times. Since our proposed operators have many zero value, 3.15 times speed up ratio can be achieved when these redundant operations are eliminated. If the approximation of DCT coefficients is used, the proposed video transcoding architecture can achieves a speed of about 3.6 to 8.4 times for transcoding the DCT coefficients to integer transform coefficients. The results are more significant for sequences with low motion activities using the approximation form of the DCT coefficients for fast computation since only a few DCT coefficients are needed to be transcoded. In addition, the proposed architecture can combine with any existing fast searching algorithm for multiple reference frame and variable block sizes estimation to speed up the transcoding process.

Table 1. Performance of the proposed transcoder, where the frame rate of the incoming bitstream was 30 frames/s. H.263 was used as the front encoder for encoding "Salesman", "Miss_America", "Hall", "Tennis", "Football" and "Flower".

\begin{tabular}{|c|c|c|c|}
\hline Sequences & $\begin{array}{c}\text { Input } \\
\text { bitrate }\end{array}$ & $\begin{array}{c}\text { Quality } \\
\text { degradation }\end{array}$ & $\begin{array}{c}\text { Speed up } \\
\text { ratio }\end{array}$ \\
\hline \multirow{2}{*}{$\begin{array}{c}\text { Salesman } \\
(352 \times 288)\end{array}$} & $512 \mathrm{k}$ & 0.03 & 8.2 \\
\cline { 2 - 4 } & $256 \mathrm{k}$ & 0.05 & 8.4 \\
\hline $\begin{array}{c}\text { Miss_America } \\
(352 \times 288)\end{array}$ & $512 \mathrm{k}$ & 0.07 & 7.9 \\
\cline { 2 - 4 } & $256 \mathrm{k}$ & 0.09 & 8.0 \\
\hline $\begin{array}{c}\text { Hall } \\
(352 \times 288)\end{array}$ & $512 \mathrm{k}$ & 0.13 & 6.9 \\
\cline { 2 - 4 } & $256 \mathrm{k}$ & 0.16 & 7.1 \\
\hline $\begin{array}{c}\text { Tennis } \\
(352 \times 240)\end{array}$ & $3 \mathrm{M}$ & 0.2 & 6.3 \\
\cline { 2 - 4 } & $1.5 \mathrm{M}$ & 0.25 & 6.4 \\
\hline $\begin{array}{c}\text { Flower } \\
(352 \times 240)\end{array}$ & $3 \mathrm{M}$ & 0.27 & 4.4 \\
\cline { 2 - 4 } & $1.5 \mathrm{M}$ & 0.3 & 4.5 \\
\hline $\begin{array}{c}\text { Football } \\
(352 \times 240)\end{array}$ & $3 \mathrm{M}$ & 0.3 & 3.6 \\
\cline { 2 - 4 } & $1.5 \mathrm{M}$ & 0.34 & 3.7 \\
\hline
\end{tabular}

\section{CONCLUSION}

In this paper, we have derived a set of operators to transcode the DCT coefficients into integer transform coefficients in the compressed domain. Using the approximation of DCT coefficients, the proposed transcoder can further speed up the transcoding process significantly with similar transcoded video quality. The proposed architecture can combine with any existing fast searching algorithm for multiple reference frames and variable block sizes estimation to speed up the transcoding process. Results of our experimental work show that the proposed architecture produces similar pictures quality with low computational complexity as compared with the conventional video transcoder.

\section{ACKNOWLEDGMENTS}

This work is supported by the Centre for Multimedia Signal Processing, Department of Electronic and Information Engineering, Hong Kong Polytechnic University and the Research Grant Council of the Hong Kong SAR Government (PolyU 5234/03E). K.T. Fung acknowledges the research studentships provided by the same University.

\section{REFERENCES}

[1] Wiegand,T., Sullivan, G.J., Bjntegaard, G. and Luthra, A., “ Overview of the H.264/AVC video coding standard" IEEE Transactions on Circuits and Systems for Video Technology, Vol.13, No.7, pp.560- 576, July 2003

[2] Video Coding for Low Bitrate Communication, ITU-T Recommendation H.263, May 1997.

[3] Jeongnam Youn, Ming-Ting Sun and Chia-Wen Lin, "Motion vector refinement for high-performance transcoding," IEEE Transactions on Multimedia, Vol.1, pp.30 - 40, March 1999

[4] G. Keeman, R. Hellinghuizen, F. Hoeksema and G. Heideman "Transcoding of MPEG bitstreams," Signal Processing: Image Communication, vol. 8, pp. 481-500, Sept. 1996.

[5] Shanableh, T.; Ghanbari, M., "Heterogeneous video transcoding to lower spatio-temporal resolutions and different encoding formats," IEEE Transactions on Multimedia, vol.2, pp.101-110, June 2000 .

[6] Bo Shen, Ishwar K. Sethi and Bhaskaran Vasudev, "Adaptive motion-vector resampling for compressed video downscaling," IEEE Transactions on circuit and systems for video technology, vol.9, no.6, September 1999.

[7] H. Sun, W. Kwok and J.W. Zdepski, "Architectures for MPEG compressed bitstream scaling,” IEEE Trans. Circuits Syst. Video Technol., vol. 6, pp. 191-199, Apr. 1996.

[8] Kai-Tat Fung and Wan-Chi Siu, "Low-Complexity H.263 to H.264 video transcoding using motion vector decomposition," paper accepted, to be published in the 2005 IEEE International Symposium on Circuits and Systems (ISCAS 2005). 